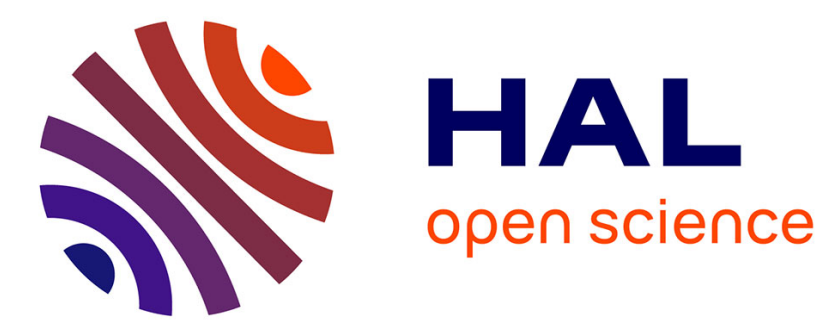

\title{
L'exploitation de l'appareil H.P.D. du Collège de France
} G. Reboul

\section{To cite this version:}

G. Reboul. L'exploitation de l'appareil H.P.D. du Collège de France. Revue de Physique Appliquée, 1969, 4 (2), pp.325-325. 10.1051/rphysap:0196900402032500 . jpa-00243284

\section{HAL Id: jpa-00243284 https://hal.science/jpa-00243284}

Submitted on 1 Jan 1969

HAL is a multi-disciplinary open access archive for the deposit and dissemination of scientific research documents, whether they are published or not. The documents may come from teaching and research institutions in France or abroad, or from public or private research centers.
L'archive ouverte pluridisciplinaire HAL, est destinée au dépôt et à la diffusion de documents scientifiques de niveau recherche, publiés ou non, émanant des établissements d'enseignement et de recherche français ou étrangers, des laboratoires publics ou privés. 


\title{
L'EXPLOITATION DE L'APPAREIL H.P.D. DU GOLLÈGE DE FRANGE
}

\author{
G. REBOUL, \\ Laboratoire de Physique Nucléaire, Collège de France.
}

\begin{abstract}
Résumé. - Le H.P.D. étant contrôlé par un petit calculateur, les tests en ligne sont réduits au minimum et l'opérateur est amené à participer à la mesure. Il dispose pour cela d'une visualisation et d'une machine à écrire connectée au calculateur, grâce à laquelle un dialogue peut s'établir. Les résultats de l'exploitation sont présentés pour la période octobre 1967novembre 1968.
\end{abstract}

Abstract. - The H.P.D. being controlled by a small computer, there are few tests on-line, and the operator must participate actively in the measurement process. A display scope and a typewriter connected to the computer establishes a man-machine dialogue. Production results from october 1967 to november 1968 are presented.

I. Organisation de la mesure. - Au Collège de France, le H.P.D. est contrôlé par un petit calculateur GDG $160 \mathrm{~A}$, dont le cycle de base est de 6,4 us. Celui-ci a un double rôle : a) un rôle de contrôle, qui consiste à commander, à partir des données de la prémesure, les diverses opérations nécessaires à la mesure : positionnement du film à la photo désirée, déplacement des chariots, mise en place du masque, etc.; b) un rôle d'acquisition de données, qui consiste à recevoir les coordonnées des points retenus et à les stocker sur bande magnétique.

Le traitement des données étant fait ultérieurement par un autre calculateur, il est intéressant et même indispensable de vérifier le plus possible de choses en ligne. Mais, à cause de la taille du calculateur, il a fallu se limiter à quelques vérifications simples et demander à l'opérateur de participer à la mesure. Les vérifications faites par le calculateur sont principalement : la croissance régulière de la coordonnée relative au chariot; la valeur de la coordonnée muette; le nombre total de points digitalisés.

L'opérateur a deux outils à sa disposition : 1) une visualisation. Deux oscilloscopes à mémoire visualisent, l'un, la photographie complète, l'autre, la photographie masquée. L'opérateur détecte ainsi immédiatement toute erreur de photographie ou de cadrage de celle-ci dans le presse-film. Ceci permet de pallier l'absence de reconnaissance du numéro binaire par le calculateur; 2) une machine à écrire connectée au calculateur. Lorsque le calculateur détecte un incident (par exemple, une erreur dans l'un des tests cités plus haut), il arrête la mesure et imprime un code d'erreur sur la machine à écrire. Il donne ainsi le contrôle à l'opérateur, qui décide de la suite à donner, en général la remesure de l'événement en cours. En particulier, les diagnostics relatifs au nombre total de points digi- talisés permettent à l'opérateur de régler le seuil de digitalisation. Lorsque l'opérateur détecte un incident (par exemple au moyen de la visualisation), il arrête la mesure en levant une clef. Le calculateur lui donne alors le contıôle en demandant son intervention par la machine à écrire.

II. Production au cours de la période octobre 196\%novembre 1968. - Le H.P.D. a travaillé $15 \mathrm{~h}$ par jour, soit $75 \mathrm{~h}$ par semaine. La figure 1 donne la production

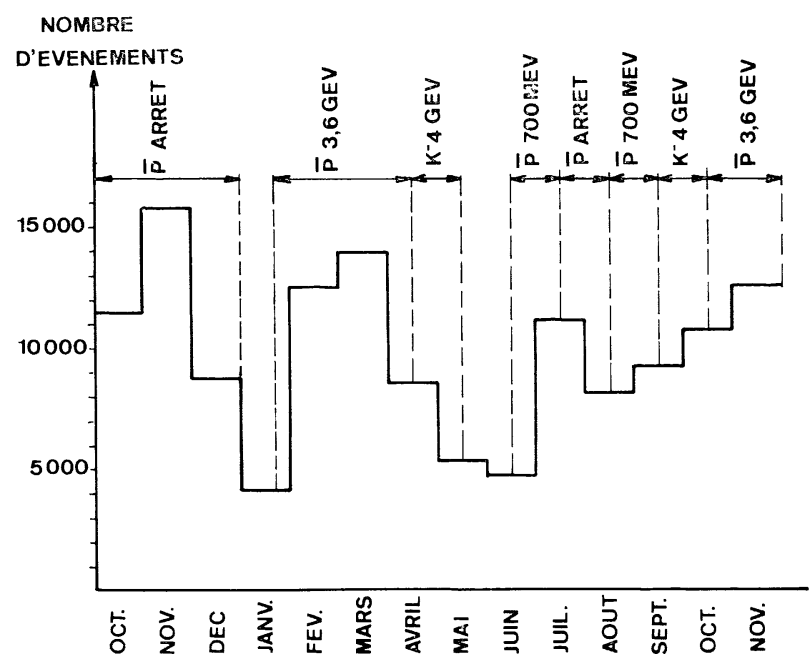

FIG. 1. - Courbe de production du H.P.D.

mensuelle entre le 1-10-67 et le 30-11-68. Le nombre total d'événements mesurés a été de 140000 . La moyenne est donc de 10000 par mois, c'est-à-dire 35 par heure. 